\title{
Blow-Up Criterion of Weak Solutions for the 3D Boussinesq Equations
}

\author{
Zhaohui Dai, ${ }^{1}$ Xiaosong Wang, ${ }^{2}$ Lingrui Zhang, ${ }^{3}$ and Wei Hou ${ }^{4}$ \\ ${ }^{1}$ College of Mathematics and Information Science, Henan Normal University, Xinxiang 453007, China \\ ${ }^{2}$ College of Information Science, Henan University of Technology, Zhengzhou 450001, China \\ ${ }^{3}$ College of Education and Teacher Development, Henan Normal University, Xinxiang 453007, China \\ ${ }^{4}$ High School Attached to HNU, Henan Normal University, Xinxiang 453007, China
}

Correspondence should be addressed to Lingrui Zhang; zhanglingrui@126.com

Received 1 July 2014; Accepted 17 September 2014

Academic Editor: Józef Banaś

Copyright (C) 2015 Zhaohui Dai et al. This is an open access article distributed under the Creative Commons Attribution License, which permits unrestricted use, distribution, and reproduction in any medium, provided the original work is properly cited.

The Boussinesq equations describe the three-dimensional incompressible fluid moving under the gravity and the earth rotation which come from atmospheric or oceanographic turbulence where rotation and stratification play an important role. In this paper, we investigate the Cauchy problem of the three-dimensional incompressible Boussinesq equations. By commutator estimate, some interpolation inequality, and embedding theorem, we establish a blow-up criterion of weak solutions in terms of the pressure $p$ in the homogeneous Besov space $\dot{B}_{\infty, \infty}^{0}$.

\section{Introduction}

This paper is devoted to establish a blow-up criterion of weak solutions to the Cauchy problem for 3-dimensional Boussinesq equations:

$$
\begin{gathered}
u_{t}+u \cdot \nabla u-\eta \Delta u+\nabla p=\theta e_{3}, \\
\theta_{t}+u \cdot \nabla \theta-v \Delta \theta=0, \\
\nabla \cdot u=0, \\
t=0: u=u_{0}(x), \quad \theta=\theta_{0}(x),
\end{gathered}
$$

where $u$ is the velocity, $p$ is the pressure, and $\theta$ is the small temperature deviations which depends on the density. $\eta \geq 0$ is the viscosity, $v \geq 0$ is called the molecular diffusivity, and $e_{3}=(0,0,1)^{T}$. The above systems describe the evolution of the velocity field $u$ for a three-dimensional incompressible fluid moving under the gravity and the earth rotation which come from atmospheric or oceanographic turbulence where rotation and stratification play an important role.
When the initial density $\theta_{0}$ is identically zero (or constant) and $\eta=0$, then (1)-(4) reduces to the classical incompressible Euler equation:

$$
\begin{gathered}
u_{t}+u \cdot \nabla u+\nabla p=0, \\
\nabla \cdot u=0, \\
\left.u(x, t)\right|_{t=0}=u_{0}(x) .
\end{gathered}
$$

From the investigation of (5), we cannot expect to have a better theory for the Boussinesq system than that of the Euler equation. For the Euler equation, a well-known criterion for the existence of global smooth solutions is the Beale-KatoMajda criterion [1]. It states that the control of the vorticity of the fluid $\omega=\operatorname{curl} u$ in $L^{1}\left(0, T ; L^{\infty}\right)$ is sufficient to get the global well posedness.

The Boussinesq equations (1)-(4) are of relevance to study a number of models coming from atmospheric or oceanographic turbulence where rotation and stratification play an important role. The scalar function $\theta$ may for instance represent temperature variation in a gravity field and $\theta e_{3}$ 
the buoyancy force. For the regularity criteria of the NavierStokes equations, we can refer to Zhou et al. [2-9], Fan and Ozawa [10], He [11], Zhang and Chen [12], and Escauriaza et al. [13].

From the mathematical point of view, the global well posedness for two-dimensional Boussinesq equations which has recently drawn much attention seems to be in a satisfactory state. More precisely, global well posedness has been shown in various function spaces and for different viscosities; we refer, for example, to [14-19]. In contrast, in the case when $\eta=v=0$, the Boussinesq system exhibits vorticity intensification and the global well-posedness issue remains an unsolved challenging open problem (except if $\theta_{0}$ is a constant, of course) which may be formally compared to the similar problem for the three-dimensional axisymmetric Euler equations with swirl.

In the three-dimensional case, there are only few results (see [20-24]). Hmidi and Rousset [23] proved the global wellposedness for the three-dimensional Euler-Boussinesq equations with axisymmetric initial data without swirl. Danchin and Paicu [20] obtained a global existence and uniqueness result for small data in Lorentz space.

Our purpose of this paper is to obtain a blow-up criterion of weak solutions in terms of Besov space.

Now, we state our result as follows.

Theorem 1. Assume that $\left(u_{0}, \theta_{0}\right) \in H^{3}\left(R^{3}\right)$ with $\operatorname{div} u_{0}=0$ in $R^{3}$. Assume that the pressure $p$ satisfies the condition

$$
\int_{0}^{T} \frac{\|\nabla p(t)\|_{\dot{B}_{\infty, \infty}^{0}}^{2 / 3}}{\left(1+\ln \left(1+\|\nabla p(t)\|_{\dot{B}_{\infty, \infty}^{0}}\right)\right)^{2 / 3}} d t<+\infty
$$

then the solution $(u, \theta)$ can be extended smoothly only up to $T$.

The paper is organized as follows. We first state some important inequalities in Section 2. We will prove Theorem 1 in Section 3.

\section{Preliminaries}

Throughout this paper, we use the following usual notations. $L^{p}\left(R^{3}\right)$ denotes the Lebesgue space and $H^{m}\left(R^{3}\right)$ denotes the standard Sobolev space. BMO denotes the space of bounded mean oscillations. $\dot{B}_{m, n}^{0}$ is the homogeneous Besov space, where $0 \leq m, n \leq+\infty$.

Lemma 2. There exists a uniform positive constant $C$, such that

$$
\begin{aligned}
\|f\|_{L^{4}}^{2} & \leq C\|f\|_{L^{2}}\|f\|_{B M O}, \\
\|f\|_{\dot{B}_{\infty, 2}^{0}} & \leq C\left(1+\|f\|_{\dot{B}_{\infty, \infty}^{0}} \ln ^{1 / 2}\left(e+\|f\|_{H^{s-1}}\right)\right)
\end{aligned}
$$

hold for all vectors $f \in H^{s-1}\left(R^{3}\right)$ with $s>5 / 2$.

Proof. See, for example, [19] or [25].
Lemma 3. From (1), one has

$$
\begin{gathered}
\|\nabla p\|_{L^{2}} \leq C\left(\|u \cdot \nabla u\|_{L^{2}}+\|\theta\|_{L^{2}}\right), \\
\|\nabla p\|_{L^{2}}^{1 / 2} \leq C\left(\|u \cdot \nabla u\|_{L^{2}}^{1 / 2}+\|\theta\|_{L^{2}}^{1 / 2}\right) .
\end{gathered}
$$

Lemma 4. Assume that $\Lambda=(-\Delta)^{1 / 2}$; one has the commutator estimate due to Kato and Ponce [24]:

$$
\begin{aligned}
& \left\|\Lambda^{s}(f g)-f \Lambda^{s} g\right\|_{L^{p}} \\
& \quad \leq C\left(\|\nabla f\|_{L^{p_{1}}}\left\|\lambda^{s-1} g\right\|_{L^{q_{1}}}+\left\|\Lambda^{s} f\right\|_{L^{p_{2}}}\|g\|_{L^{q_{2}}}\right),
\end{aligned}
$$

with $s>0,1 / p=1 / p_{1}+1 / q_{1}=1 / p_{2}+1 / q_{2}$.

Lemma 5 (the Gagliardo-Nirenberg inequality). Consider

$$
\begin{gathered}
\|\nabla f\|_{L^{4}} \leq C\|f\|_{L^{4}}^{1 / 5}\|\Delta f\|_{L^{2}}^{4 / 5}, \\
\|\nabla f\|_{L^{3}} \leq C\|\nabla f\|_{L^{2}}^{3 / 4}\left\|\Lambda^{3} f\right\|_{L^{2}}^{1 / 4}, \\
\left\|\Lambda^{3} f\right\|_{L^{3}} \leq C\|\nabla f\|_{L^{2}}^{1 / 6}\left\|\Lambda^{4} f\right\|_{L^{2}}^{5 / 6} .
\end{gathered}
$$

\section{Proof of Theorem 1}

Proof of Theorem 1. Multiplying (1) by $u$, using (3), and integrating in $R^{3}$, we derive

$$
\begin{aligned}
& \frac{1}{2} \frac{d}{d t}\|u\|_{L^{2}}^{2}+\eta\|\nabla u\|_{L^{2}}^{2} \\
& \quad=\int_{R^{3}} \theta e_{3} \cdot u d x \leq\|\theta\|_{L^{2}}\|u\|_{L^{2}} \\
& \quad \leq \frac{1}{2}\|\theta\|_{L^{2}}^{2}+\frac{1}{2}\|u\|_{L^{2}}^{2} .
\end{aligned}
$$

Multiplying (2) by $\theta$, using (3), and integrating in $R^{3}$, we obtain

$$
\frac{1}{2} \frac{d}{d t}\|\theta\|_{L^{2}}^{2}+v\|\nabla \theta\|_{L^{2}}=0
$$

Combining (14) and (15), using the Gronwall inequality, we deduce that

$$
\begin{gathered}
\|u\|_{L^{\infty}\left(0, T ; L^{2}\right)}+\|u\|_{L^{2}\left(0, T ; H^{1}\right)} \leq C, \\
\|\theta\|_{L^{\infty}\left(0, T ; L^{2}\right)}+\|\theta\|_{L^{2}\left(0, T ; H^{1}\right)} \leq C .
\end{gathered}
$$


Multiplying (1) by $|u|^{2} u$, using (3) and (7), and integrating in $R^{3}$, we derive

$$
\begin{aligned}
\int\left[|u|^{2} \cdot u\left(u_{t}+u \cdot \nabla u-\eta \Delta u+\nabla p\right)\right] \\
=\frac{1}{4} \frac{d}{d t} \int|u|^{4} d x+\int|u|^{2} \cdot u^{2} \cdot \nabla u d x \\
\quad+\frac{\eta}{2} \int\left(\nabla|u|^{2}\right)^{2} d x \\
\quad+\eta \int|u|^{2}|\nabla u|^{2} d x+\int(u \cdot \nabla p)|u|^{2} d x \\
=\int|u|^{2} \cdot u \cdot \theta e_{3} d x \\
\leq C \int\left(|u|^{4}+|\theta|^{4}\right) d x
\end{aligned}
$$

that is,

$$
\begin{aligned}
& \frac{1}{4} \frac{d}{d t} \int|u|^{4} d x+\frac{\eta}{2} \int\left(\nabla|u|^{2}\right)^{2} d x+\eta \int|u|^{2}|\nabla u|^{2} d x \\
& \quad \leq-\int(u \cdot \nabla p)|u|^{2} d x+C \int|u|^{4}+|\theta|^{4} d x \\
& \quad \leq\|u\|_{L^{4}}^{3}\|\nabla p\|_{L^{4}}+C\|u\|_{L^{4}}^{4}+C\|\theta\|_{L^{4}}^{4} \\
& \quad \leq C\|u\|_{L^{4}}^{3}\|\nabla p\|_{L^{2}}^{1 / 2}\|\nabla p\|_{\mathrm{BMO}}^{1 / 2}+C\|u\|_{L^{4}}^{4}+C\|\theta\|_{L^{4}}^{4} .
\end{aligned}
$$

Multiplying (2) by $|\theta|^{2} \theta$, using (3), and integrating in $R^{3}$, we arrive at

$$
\begin{aligned}
\int\left(|\theta|^{2} \cdot \theta \cdot \theta_{t}+|\theta|^{2} \theta \cdot u \cdot \nabla \theta-v|\theta|^{2} \cdot \theta \cdot \Delta \theta\right) d x \\
=\frac{1}{4} \frac{d}{d t} \int|\theta|^{4} d x+v \int|\theta|^{2}(\nabla \theta)^{2} d x \\
\quad+\frac{v}{2} \int|\theta|^{2}(\operatorname{div} \theta)^{2} d x
\end{aligned}
$$

Combining (18) and (19), using (9) and (16), we derive that

$$
\begin{aligned}
\frac{1}{4} \frac{d}{d t} \int\left(|\theta|^{4}+|u|^{4}\right) d x+\frac{\eta}{2} \int\left(\nabla|u|^{2}\right)^{2} d x \\
\quad+\eta \int|u|^{2}|\nabla u|^{2} d x+v \int|\theta|^{2}(\nabla \theta)^{2} d x \\
\quad+\frac{v}{2} \int|\theta|^{2}(\operatorname{div} \theta)^{2} d x \\
\leq C\|u\|_{L^{4}}^{3}\|\nabla p\|_{L^{2}}^{1 / 2}\|\nabla p\|_{\mathrm{BMO}}^{1 / 2}+C\|u\|_{L^{4}}^{4}+C\|\theta\|_{L^{4}}^{4} \\
\leq C\|u\|_{L^{4}}^{3}\left(\|u \cdot \nabla u\|_{L^{2}}^{1 / 2}+\|\theta\|_{L^{2}}^{1 / 2}\right)\|\nabla p\|_{\mathrm{BMO}}^{1 / 2} \\
\quad+C\|u\|_{L^{4}}^{4}+C\|\theta\|_{L^{4}}^{4} \\
\leq 2 C\|u\|_{L^{4}}^{4}\|\nabla p\|_{\mathrm{BMO}}^{2 / 3}+\frac{\eta}{2}\||u| \nabla u\|_{L^{2}}^{2} \\
\quad+C\|\theta\|_{L^{2}}^{2}+C\|u\|_{L^{4}}^{4}+C\|\theta\|_{L^{4}}^{4},
\end{aligned}
$$

which implies

$$
\begin{aligned}
& \frac{d}{d t} \int\left(|\theta|^{4}+|u|^{4}\right) d x+\eta \int\left(\nabla|u|^{2}\right)^{2} d x \\
& +\eta \int|u|^{2}|\nabla u|^{2} d x+v \int|\theta|^{2}(\nabla \theta)^{2} d x \\
& +v \int|\theta|^{2}(\operatorname{div} \theta)^{2} d x \\
& \leq 8 C\|u\|_{L^{4}}^{4}\|\nabla p\|_{\mathrm{BMO}}^{2 / 3}+4 C\|\theta\|_{L^{2}}^{2} \\
& +4 C\|u\|_{L^{4}}^{4}+4 C\|\theta\|_{L^{4}}^{4} \\
& \leq 8 C\|u\|_{L^{4}}^{4}\|\nabla p\|_{\dot{B}_{\infty, \infty}^{0}}^{2 / 3} \ln ^{1 / 3}\left(1+\|\nabla p\|_{H^{2}}\right) \\
& +4 C\|\theta\|_{L^{2}}^{2}+4 C\|u\|_{L^{4}}^{4}+4 C\|\theta\|_{L^{4}}^{4} \\
& \leq 8 C\|u\|_{L^{4}}^{4}\|\nabla p\|_{\dot{B}_{\infty, \infty}^{0}}^{2 / 3} \\
& \times \ln ^{1 / 3}\left(1+\|\nabla \Delta u\|_{L^{2}}+\|\Delta \theta\|_{L^{2}}\right) \\
& +4 C\|\theta\|_{L^{2}}^{2}+4 C\|u\|_{L^{4}}^{4}+4 C\|\theta\|_{L^{4}}^{4} \\
& \leq 8 C\|u\|_{L^{4}}^{4} \frac{\|\nabla p\|_{\dot{B}_{\infty, \infty}^{0}}^{2 / 3}}{\left(1+\ln \left(1+\|\nabla p\|_{\dot{B}_{\infty, \infty}^{0}}\right)\right)^{2 / 3}} \\
& \times \ln \left(1+\|\nabla \Delta u\|_{L^{2}}+\|\Delta \theta\|_{L^{2}}\right) \\
& +4 C\|\theta\|_{L^{2}}^{2}+4 C\|u\|_{L^{4}}^{4}+4 C\|\theta\|_{L^{4}}^{4} .
\end{aligned}
$$

Choosing $t \in\left[T_{*}, T\right]$ and setting

$$
y(t)=\sup _{t \in\left[T_{*}, T\right]}\left(\|\nabla \cdot \Delta u(t)\|_{L^{2}}+\|\Delta \theta\|_{L^{2}}\right)
$$

we have

$$
\sup _{t \in\left[T_{*}, T\right]}\left(\|u\|_{L^{4}}+\|\theta\|_{L^{4}}\right) \leq C_{*}(1+y(t))^{C \varepsilon},
$$

where $\varepsilon$ is a small enough constant, such that

$$
\int_{T_{*}}^{T} \frac{\|\nabla p\|_{\dot{B}_{\infty, \infty}^{0}}^{2 / 3}}{\left(1+\ln \left(1+\|\nabla p\|_{\dot{B}_{\infty, \infty}^{0}}\right)\right)^{2 / 3}} d t<\varepsilon .
$$

Next, we want to estimate the $L^{2}$-norm of $\nabla u$ and $\nabla \theta$.

Multiplying (1) by $-\Delta u$, integrating in $R^{3}$, and using (3) and (11), we derive that

$$
\begin{gathered}
\int u_{t} \cdot(-\Delta u) d x+\int(u \cdot \nabla u)(-\Delta u) d x \\
+\eta\|\Delta u\|_{L^{2}}^{2}+\int \nabla p \cdot(-\Delta u) d x \\
=-\int \theta e_{3} \cdot \Delta u d x
\end{gathered}
$$


that is,

$$
\begin{aligned}
& \frac{1}{2} \frac{d}{d t} \int|\nabla u|^{2} d x+\eta \int|\Delta u|^{2} d x \\
& \quad=\int(u \cdot \nabla u) \Delta u d x-\int \theta e_{3} \Delta u d x \\
& \quad \leq\|u\|_{L^{4}}\|\nabla u\|_{L^{4}}\|\Delta u\|_{L^{2}}+\|\Delta u\|_{L^{2}}\|\theta\|_{L^{2}} \\
& \quad \leq C\|u\|_{L^{4}}\|u\|_{L^{4}}^{1 / 5}\|\Delta u\|_{L^{2}}^{4 / 5}\|\Delta u\|_{L^{2}}+\frac{\eta}{4}\|\Delta u\|_{L^{2}}^{2}+C\|\theta\|_{L^{2}}^{2} \\
& \quad \leq \frac{\eta}{4}\|\Delta u\|_{L^{2}}^{2}+C\|u\|_{L^{4}}^{12}+\frac{\eta}{4}\|\Delta u\|_{L^{2}}^{2}+C\|\theta\|_{L^{2}}\|\Delta \theta\|_{L^{2}} \\
& \quad \leq \frac{\eta}{2}\|\Delta u\|_{L^{2}}^{2}+C\|u\|_{L^{4}}^{12}+\frac{v}{4}\|\Delta \theta\|_{L^{2}}^{2}+C\|\theta\|_{L^{2}}^{2} .
\end{aligned}
$$

Multiplying (2) by $-\Delta \theta$, integrating in $R^{3}$, and using (3) and (11), we derive that

$$
\int \theta_{t} \cdot(-\Delta \theta) d x+\int(u \cdot \nabla \theta)(-\Delta \theta) d x+v\|\Delta \theta\|_{L^{2}}^{2}=0 ;
$$

that is,

$$
\begin{aligned}
& \frac{1}{2} \frac{d}{d t} \int|\nabla \theta|^{2} d x+v \int|\Delta \theta|^{2} d x \\
& \quad=\int(u \cdot \nabla \theta) \Delta \theta+\frac{\eta}{4}\|\Delta u\|_{L^{2}}^{2}+C\|\theta\|_{L^{2}}^{2} d x \\
& \quad \leq\|u\|_{L^{4}}\|\nabla \theta\|_{L^{4}}\|\Delta \theta\|_{L^{2}} \\
& \quad \leq C\|u\|_{L^{4}}\|\theta\|_{L^{4}}^{1 / 5}\|\Delta \theta\|_{L^{2}}^{4 / 5}\|\Delta \theta\|_{L^{2}} \\
& \quad \leq \frac{v}{4}\|\Delta \theta\|_{L^{2}}^{2}+C\|u\|_{L^{4}}^{10}\|\theta\|_{L^{4}}^{2} .
\end{aligned}
$$

Combining (26) and (28), using (16), we deduce

$$
\begin{aligned}
& \frac{1}{2} \frac{d}{d t} \int\left(|\nabla u|^{2}+|\nabla \theta|^{2}\right) d x+\eta \int|\Delta u|^{2} d x+v \int|\Delta \theta|^{2} d x \\
& \leq \frac{\eta}{2}\|\Delta u\|_{L^{2}}^{2}+C\|u\|_{L^{4}}^{12}+\frac{v}{4}\|\Delta \theta\|_{L^{2}}^{2}+C\|\theta\|_{L^{2}}^{2} \\
& \quad+\frac{v}{4}\|\Delta \theta\|_{L^{2}}^{2}+C\|u\|_{L^{4}}^{10}\|\theta\|_{L^{4}}^{2} \\
& =\frac{\eta}{2}\|\Delta u\|_{L^{2}}^{2}+\frac{v}{2}\|\Delta \theta\|_{L^{2}}^{2}+C\|u\|_{L^{4}}^{12}+C\|\theta\|_{L^{2}}^{2} \\
& \quad+C\|u\|_{L^{4}}^{10}\|\theta\|_{L^{4}}^{2} ;
\end{aligned}
$$

that is,

$$
\begin{aligned}
& \frac{d}{d t} \int\left(|\nabla u|^{2}+|\nabla \theta|^{2}\right) d x+\eta \int|\Delta u|^{2} d x+v \int|\Delta \theta|^{2} d x \\
& \quad \leq 2 C\|u\|_{L^{4}}^{12}+2 C\|\theta\|_{L^{2}}^{2}+2 C\|u\|_{L^{4}}^{10}\|\theta\|_{L^{4}}^{2},
\end{aligned}
$$

which implies that

$$
\|\nabla u(t, \cdot)\|_{L^{2}}^{2}+\|\nabla \theta(t, \cdot)\|_{L^{2}}^{2} \leq C(1+y(t))^{C \varepsilon} .
$$

Last, we will estimate the $H^{3}$-norm and $H^{4}$-norm of $u$ and $\theta$ and use the operator $\Lambda$ to derive our goal.

Applying $\Lambda^{3}=(-\Delta)^{3 / 2}$ to (1) and then multiplying (1) with $\Lambda^{3} u$, we deduce

$$
\begin{aligned}
& \int \Lambda^{3} u_{t} \cdot \Lambda^{3} u d x+\int \Lambda^{3}(u \cdot \nabla u) \cdot \Lambda^{3} u d x \\
& \quad-\eta \int \Lambda^{3} \Delta u \cdot \Lambda^{3} u d x+\int \Lambda^{3} \nabla p\left(\Lambda^{3} u\right) d x \\
& =\int \Lambda^{3} \theta e_{3} \cdot \Lambda^{3} u d x
\end{aligned}
$$

that is,

$\frac{1}{2} \frac{d}{d t} \int\left|\Lambda^{3} u\right|^{2} d x+\eta \int\left|\Lambda^{4} u\right|^{2} d x$

$$
\begin{aligned}
& =-\int\left[\Lambda^{3}(u \cdot \nabla u)-u \cdot \nabla \Lambda^{3} u\right] \cdot \Lambda^{3} u d x+\int \Lambda^{3} \theta e_{3} \Lambda^{3} u d x \\
& \leq C\left(\|\nabla u\|_{L^{3}}\left\|\Lambda^{3} u\right\|_{L^{3}}^{2}+\left\|\Lambda^{3} u\right\|_{L^{2}}\left\|\Lambda^{4} u\right\|_{L^{2}}\right)+\left\|\Lambda^{3} u\right\|_{L^{2}}\|\theta\|_{L^{2}}
\end{aligned}
$$$$
\leq C\|\nabla u\|_{L^{2}}^{3 / 4}\left\|\Lambda^{3} u\right\|_{L^{2}}^{1 / 4}\|\nabla u\|_{L^{2}}^{1 / 3}\left\|\Lambda^{4} u\right\|_{L^{2}}^{5 / 3}
$$$$
+\left\|\Lambda^{3} u\right\|_{L^{2}}\|\theta\|_{L^{2}}+C\left\|\Lambda^{3} u\right\|_{L^{2}}^{2}+\frac{\eta}{16}\left\|\Lambda^{4} u\right\|_{L^{2}}
$$$$
\leq C\|\nabla u\|_{L^{2}}^{13 / 12}\left\|\Lambda^{3} u\right\|_{L^{2}}^{1 / 4}\left\|\Lambda^{4} u\right\|_{L^{2}}^{5 / 3}
$$$$
+C\left\|\Lambda^{3} u\right\|_{L^{2}}^{2}+C\|\theta\|_{L^{2}}+C\left\|\Lambda^{3} u\right\|_{L^{2}}^{2}+\frac{\eta}{16}\left\|\Lambda^{4} u\right\|_{L^{2}}
$$$$
\leq \frac{\eta}{16}\left\|\Lambda^{4} u\right\|_{L^{2}}+C\|\nabla u\|_{L^{2}}^{13 / 10}\left\|\Lambda^{3} u\right\|_{L^{2}}^{1 / 2}
$$$$
+2 C\left\|\Lambda^{3} u\right\|_{L^{2}}^{2}+C\|\theta\|_{L^{2}}+\frac{\eta}{16}\left\|\Lambda^{4} u\right\|_{L^{2}}
$$$$
\leq C\|\nabla u\|_{L^{2}}^{13 / 12}\left\|\Lambda^{3} u\right\|_{L^{2}}^{1 / 4}\left\|\Lambda^{4} u\right\|_{L^{2}}^{5 / 3}+C\left\|\Lambda^{3} u\right\|_{L^{2}}^{2}
$$$$
+C\|\theta\|_{L^{2}}+C\left\|\Lambda^{3} u\right\|_{L^{2}}^{2}+\frac{\eta}{8}\left\|\Lambda^{4} u\right\|_{L^{2}}
$$$$
=\frac{\eta}{4}\left\|\Lambda^{4} u\right\|_{L^{2}}^{2}+C\|\nabla u\|_{L^{2}}^{13 / 10}\left\|\Lambda^{3} u\right\|_{L^{2}}^{1 / 2}
$$$$
+2 C\left\|\Lambda^{3} u\right\|_{L^{2}}^{2}+C\|\theta\|_{L^{2}} .
$$

Similarly, applying $\Lambda^{3}$ to (2) and multiplying (2) by $\Lambda^{3} \theta$, we derive

$$
\begin{gathered}
\int \Lambda^{3} \theta_{t}\left(\Lambda^{3} \theta\right) d x+\int \Lambda^{3}(u \cdot \nabla \theta) \Lambda^{3} \theta d x \\
-v \int \Lambda^{3} \Delta \theta \cdot \Lambda^{3} \theta d x=0 ;
\end{gathered}
$$


that is,

$$
\begin{aligned}
\frac{1}{2} \frac{d}{d t} \int\left|\Lambda^{3} \theta\right|^{2} d x+v \int\left|\Lambda^{4} \theta\right|^{2} d x \\
=-\int \Lambda^{3}(u \cdot \nabla \theta) \Lambda^{3} \theta d x \\
=C\|\nabla u\|_{L^{3}}\left\|\Lambda^{3} \theta\right\|_{L^{3}}^{2}+\left\|\Lambda^{3} u\right\|_{L^{3}}\|\nabla \theta\|_{L^{3}}\left\|\Lambda^{3} \theta\right\|_{L^{3}} \\
\leq C\|\nabla u\|_{L^{2}}^{3 / 4}\left\|\Lambda^{3} u\right\|_{L^{2}}^{1 / 4}\|\nabla \theta\|_{L^{2}}^{1 / 3}\left\|\Lambda^{4} \theta\right\|_{L^{2}}^{5 / 3} \\
\quad+C\|\nabla u\|_{L^{2}}^{1 / 6}\left\|\Lambda^{4} u\right\|_{L^{2}}^{5 / 6}\|\nabla \theta\|_{L^{2}}^{3 / 4}\left\|\Lambda^{3} \theta\right\|_{L^{2}}^{1 / 4} \\
\quad \times\|\nabla \theta\|_{L^{2}}^{1 / 6}\left\|\Lambda^{4} \theta\right\|_{L^{2}}^{5 / 6} \\
\leq C\|\nabla u\|_{L^{2}}^{9 / 2}\left\|\Lambda^{3} u\right\|_{L^{2}}^{3 / 2}\|\nabla \theta\|_{L^{2}}^{2}+\frac{\nu}{4}\left\|\Lambda^{4} \theta\right\|_{L^{2}}^{2} \\
\quad+C\|\nabla u\|_{L^{2}}^{1 / 3}\left\|\Lambda^{4} u\right\|_{L^{2}}^{5 / 3} \\
\quad+C\|\nabla \theta\|_{L^{2}}^{3 / 2}\left\|\Lambda^{3} \theta\right\|_{L^{2}}^{1 / 2}\|\nabla \theta\|_{L^{2}}^{1 / 3}\left\|\Lambda^{4} \theta\right\|_{L^{2}}^{5 / 3} \\
\leq C\|\nabla u\|_{L^{2}}^{9 / 2}\left\|\Lambda^{3} u\right\|_{L^{2}}^{3 / 2}\|\nabla \theta\|_{L^{2}}^{2}+\frac{v}{4}\left\|\Lambda^{4} \theta\right\|_{L^{2}}^{2} \\
\quad+C\|\nabla u\|_{L^{2}}^{2}+\frac{\eta}{4}\left\|\Lambda^{4} u\right\|_{L^{2}}^{2} \\
\quad+C\|\nabla \theta\|_{L^{2}}^{9}\left\|\Lambda^{3} \theta\right\|_{L^{2}}^{3}\|\nabla \theta\|_{L^{2}}^{2}+\frac{v}{4}\left\|\Lambda^{4} \theta\right\|_{L^{2}}^{2}
\end{aligned}
$$

Combining (33) and (35), we have

$$
\begin{aligned}
& \frac{1}{2} \frac{d}{d t}\left(\int\left|\Lambda^{3} u\right|^{2}\left|\Lambda^{3} \theta\right|^{2} d x\right) \\
& \quad+\eta \int\left|\Lambda^{4} u\right|^{2} d x+v \int\left|\Lambda^{4} \theta\right|^{2} d x \\
& \leq \frac{\eta}{2}\left\|\Lambda^{4} u\right\|_{L^{2}}^{2}+C\|\nabla u\|_{L^{2}}^{13 / 10}\left\|\Lambda^{3} u\right\|_{L^{2}}^{1 / 2} \\
& \quad+2 C\left\|\Lambda^{3} u\right\|_{L^{2}}^{2}+C\|\theta\|_{L^{2}} \\
& \quad+C\|\nabla u\|_{L^{2}}^{9 / 2}\left\|\Lambda^{3} u\right\|_{L^{2}}^{3 / 2}\|\nabla \theta\|_{L^{2}}^{2}+\frac{v}{2}\left\|\Lambda^{4} \theta\right\|_{L^{2}}^{2} \\
& \quad+C\|\nabla u\|_{L^{2}}^{2}+C\|\nabla \theta\|_{L^{2}}^{9}\left\|\Lambda^{3} \theta\right\|_{L^{2}}^{3}\|\nabla \theta\|_{L^{2}}^{2}
\end{aligned}
$$

that is,

$$
\begin{aligned}
& \frac{d}{d t}\left(\int\left|\Lambda^{3} u\right|^{2}\left|\Lambda^{3} \theta\right|^{2} d x\right) \\
& \quad+\eta \int\left|\Lambda^{4} u\right|^{2} d x+v \int\left|\Lambda^{4} \theta\right|^{2} d x \\
& \leq 2 C\|\nabla u\|_{L^{2}}^{13 / 10}\left\|\Lambda^{3} u\right\|_{L^{2}}^{1 / 2} \\
& \quad+4 C\left\|\Lambda^{3} u\right\|_{L^{2}}^{2}+2 C\|\theta\|_{L^{2}}
\end{aligned}
$$

$$
\begin{aligned}
& +2 C\|\nabla u\|_{L^{2}}^{9 / 2}\left\|\Lambda^{3} u\right\|_{L^{2}}^{3 / 2}\|\nabla \theta\|_{L^{2}}^{2}+2 C\|\nabla u\|_{L^{2}}^{2} \\
& +2 C\|\nabla \theta\|_{L^{2}}^{9}\left\|\Lambda^{3} \theta\right\|_{L^{2}}^{3}\|\nabla \theta\|_{L^{2}}^{2} .
\end{aligned}
$$

Choosing $\varepsilon$ small enough, using (16), (23), and (24), we conclude that

$$
\begin{gathered}
\|u\|_{L^{\infty}\left(0, T ; H^{3}\right)}+\|u\|_{L^{2}\left(0, T ; H^{4}\right)} \leq C, \\
\|\theta\|_{L^{\infty}\left(0, T ; H^{3}\right)}+\|\theta\|_{L^{2}\left(0, T ; H^{4}\right)} \leq C .
\end{gathered}
$$

We complete the proof.

\section{Conflict of Interests}

The authors declare that they have no competing interests.

\section{Authors' Contribution}

The authors declare that the work was realized in collaboration with the same responsibility. All authors read and approved the final paper.

\section{Acknowledgments}

All authors thank the reviewers for their suggestions; this paper was in part supported by the NNSF of China (nos. 11326154 and U1304101), the NSFC Projects of Education Department of Henan Province (no. 14B110029), and the Program for Science and Technology Innovation Talents in University of Henan Province (no. 13HASTIT040).

\section{References}

[1] J. T. Beale, T. Kato, and A. Majda, "Remarks on the breakdown of smooth solutions for the 3-D Euler equations," Communications in Mathematical Physics, vol. 94, no. 1, pp. 61-66, 1984.

[2] Y. Zhou, "Regularity criteria in terms of pressure for the 3-D Navier-Stokes equations in a generic domain," Mathematische Annalen, vol. 328, no. 1-2, pp. 173-192, 2004.

[3] Y. Zhou, "On regularity criteria in terms of pressure for the Navier-Stokes equations in $\mathbb{R}^{3}$," Proceedings of the American Mathematical Society, vol. 134, no. 1, pp. 149-156, 2006.

[4] Y. Zhou, "On a regularity criterion in terms of the gradient of pressure for the Navier-Stokes equations in $R^{N}$," Zeitschrift für Angewandte Mathematik und Physik: ZAMP, vol. 57, no. 3, pp. 384-392, 2006.

[5] Y. Zhou and S. Gala, "Logarithmically improved regularity criteria for the Navier-Stokes equations in multiplier spaces," Journal of Mathematical Analysis and Applications, vol. 356, no. 2, pp. 498-501, 2009.

[6] Y. Zhou, "A new regularity criterion for the Navier-Stokes equations in terms of the gradient of one velocity component," Methods and Applications of Analysis, vol. 9, no. 4, pp. 563-578, 2002.

[7] Y. Zhou, "A new regularity criterion for weak solutions to the Navier-Stokes equations," Journal de Mathématiques Pures et Appliquées, vol. 84, no. 11, pp. 1496-1514, 2005. 
[8] Y. Zhou and M. Pokorny, "On a regularity criterion for the Navier -Stokes equations involving gradient of one velocity component," Journal of Mathematical Physics, vol. 50, Article ID 123514, pp. 1-11, 2009.

[9] Y. Zhou and M. Pokorny, "On the regularity of the solutions of the Navier-Stokes equations via one velocity component," Nonlinearity, vol. 23, no. 5, pp. 1097-1107, 2010.

[10] J. Fan and T. Ozawa, "Regularity criterion for weak solutions to the Navier-Stokes equations in terms of the gradient of the pressure," Journal of Inequalities and Applications, vol. 2008, Article ID 412678, 6 pages, 2008.

[11] C. He, "New sufficient conditions for regularity of solutions to the Navier-Stokes equations," Advances in Mathematical Sciences and Applications, vol. 12, no. 2, pp. 535-548, 2002.

[12] Z. Zhang and Q. Chen, "Regularity criterion via two components of vorticity on weak solutions to the Navier-Stokes equations in $\mathbb{R}^{3}$," Journal of Differential Equations, vol. 216, no. 2, pp. 470-481, 2005.

[13] L. Escauriaza, G. A. Seregin, and V. Sverak, " $\mathrm{L}^{3, \infty}$-solutions of NavierStokes equations and Backward Uniqueness," Russian Mathematical Surveys, vol. 58, pp. 211-250, 2003, translated from Uspekhi Matematicheskikh Nauk, vol.58, pp. 3-44, 2003.

[14] H. Abidi and T. Hmidi, "On the global well-posedness for Boussinesq system," Journal of Differential Equations, vol. 233, no. 1, pp. 199-220, 2007.

[15] J. R. Cannon and E. Dibenedetto, "The initial value problem for the Boussinesq equations with data in $L^{p}$," in Approximation Methods for Navier-Stokes Problems, vol. 771 of Lecture Notes in Mathematics, pp. 129-144, Springer, Berlin, Germany, 1980.

[16] D. Chae, "Global regularity for the 2D Boussinesq equations with partial viscosity terms," Advances in Mathematics, vol. 203, no. 2, pp. 497-513, 2006.

[17] R. Danchin and M. Paicu, "Global well-posedness issues for the inviscid Boussinesq system with Yudovich's type data," Communications in Mathematical Physics, vol. 290, no. 1, pp. 1-14, 2009.

[18] T. Hmidi and S. Keraani, "On the global well-posedness of the Boussinesq system with zero viscosity," Indiana University Mathematics Journal, vol. 58, no. 4, pp. 1591-1618, 2009.

[19] T. Y. Hou and C. Li, "Global well-posedness of the viscous Boussinesq equations," Discrete and Continuous Dynamical Systems. Series A, vol. 12, no. 1, pp. 1-12, 2005.

[20] R. Danchin and M. Paicu, "Existence and uniqueness results for the Boussinesq system with data in Lorentz spaces," Physica D: Nonlinear Phenomena, vol. 237, no. 10-12, pp. 1444-1460, 2008.

[21] J. Fan and Y. Zhou, "A note on regularity criterion for the 3D Boussinesq system with partial viscosity," Applied Mathematics Letters, vol. 22, no. 5, pp. 802-805, 2009.

[22] T. Hmidi and F. Rousset, "Global well-posedness for the NavierStokes-Boussinesq system with axisymmetric data," Annales de l'Institut Henri Poincare (C) Non Linear Analysis, vol. 27, no. 5, pp. 1227-1246, 2009.

[23] T. Hmidi and F. Rousset, "Global well-posedness for the EulerBoussinesq system with axisymmetric data," Journal of Functional Analysis, vol. 260, no. 3, pp. 745-796, 2010.

[24] T. Kato and G. Ponce, "Commutator estimates and the Euler and Navier-Stokes equations," Communications on Pure and Applied Mathematics, vol. 41, no. 7, pp. 891-907, 1988.

[25] H. Kozono and Y. Taniuchi, "Bilinear estimates in BMO and the Navier-Stokes equations," Mathematische Zeitschrift, vol. 235, no. 1, pp. 173-194, 2000. 


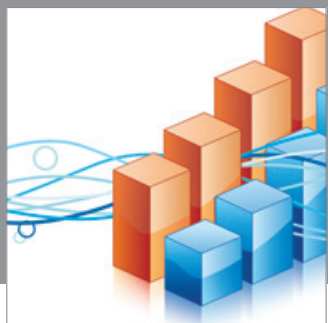

Advances in

Operations Research

mansans

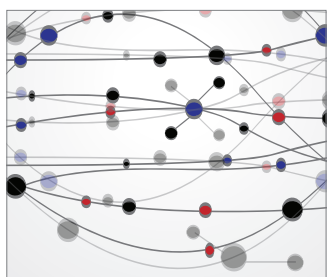

The Scientific World Journal
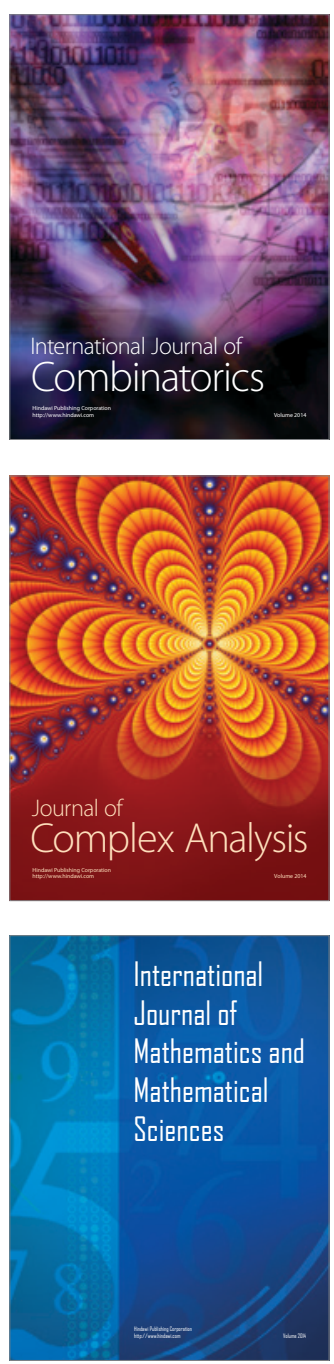
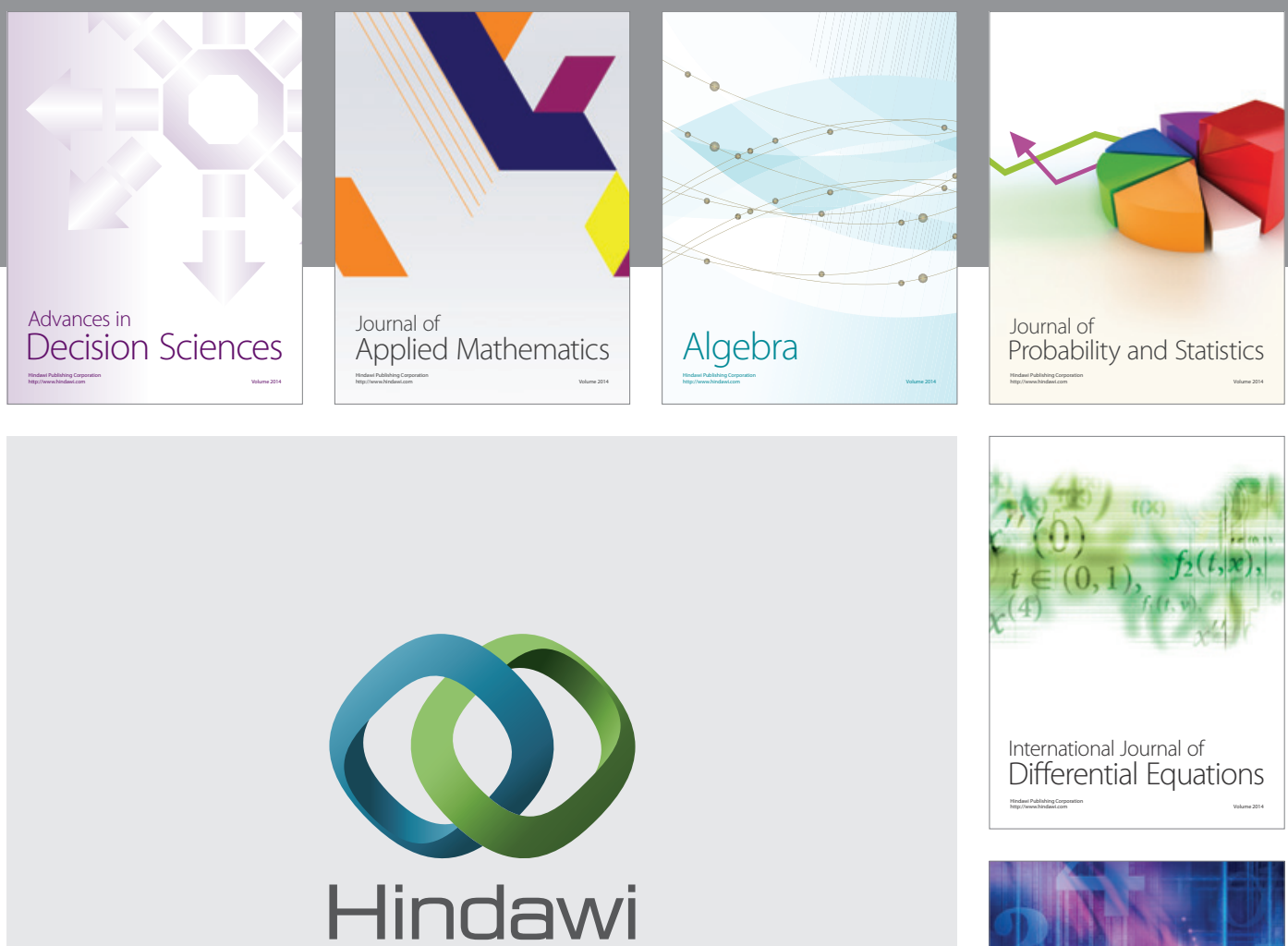

Submit your manuscripts at http://www.hindawi.com
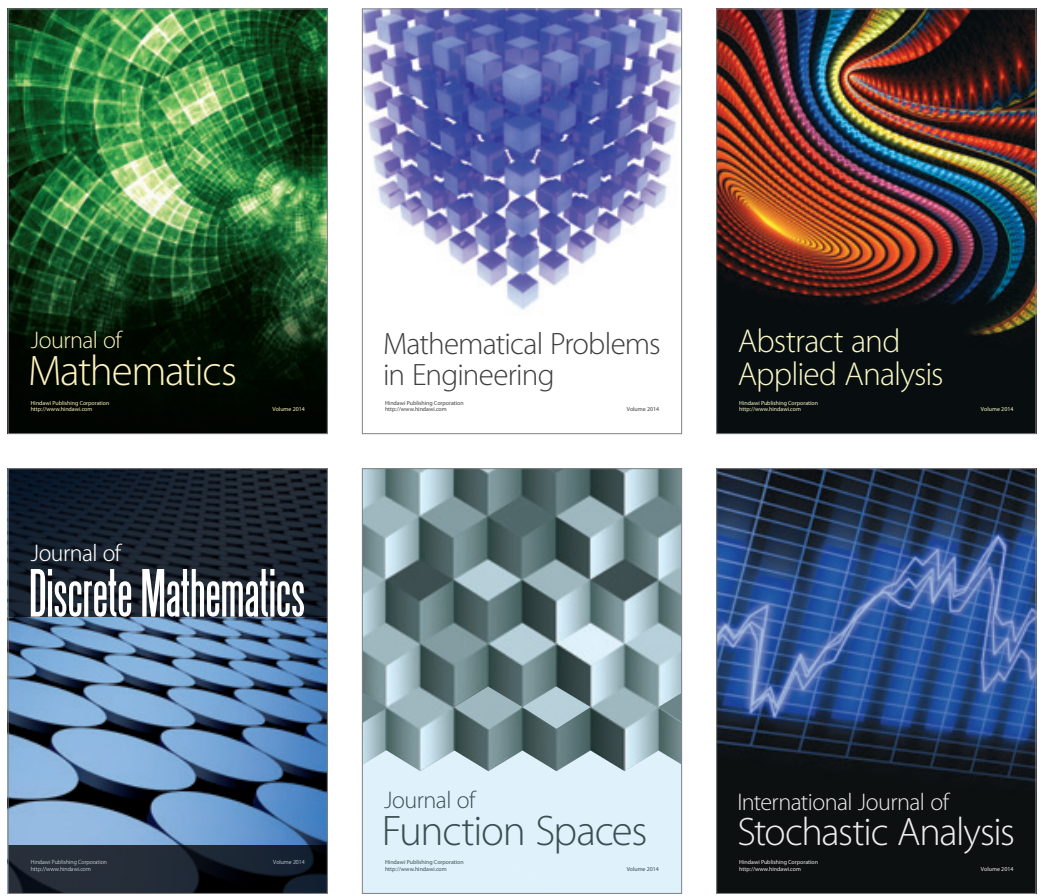

Journal of

Function Spaces

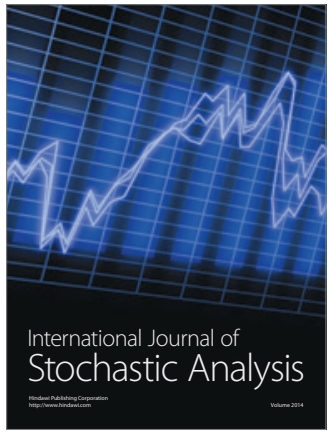

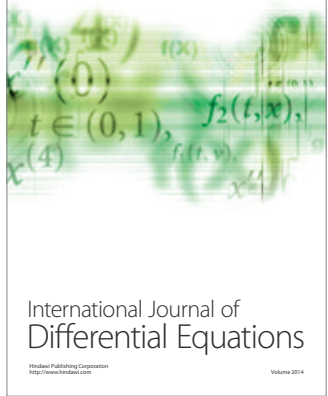
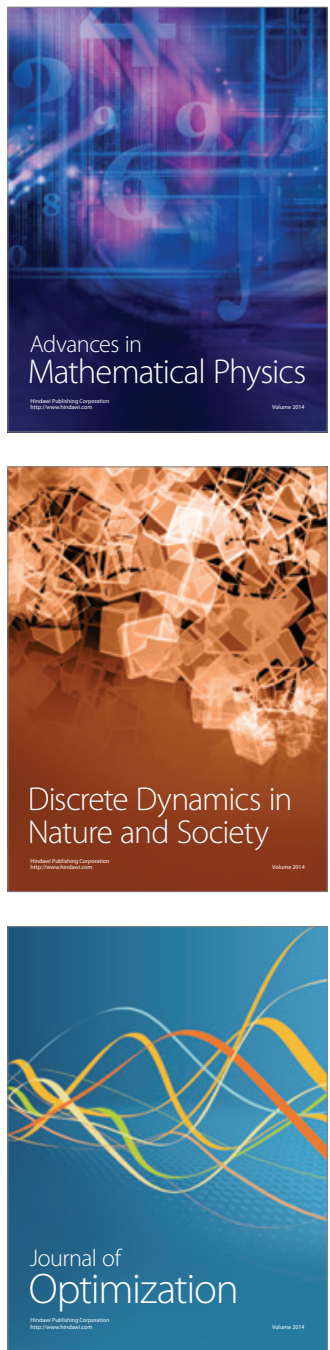